\title{
$\beta$-Cyclodextrin Interaction with $N$-Hexanoyl Homoserine Lactone as Quorum Sensing Signal Produced in Gram-Negative Bacteria
}

\author{
Chigusa Okano ${ }^{1,2}$, Marina Arai ${ }^{1,2}$, Eri Nasuno ${ }^{1,2}$, Ken-ichi Iimura ${ }^{1,2}$, Tomohiro Morohoshi ${ }^{1,2}$, \\ Tsukasa Ikeda ${ }^{1,2}$ and Norihiro Kato ${ }^{1,2, *}$ \\ ${ }^{1}$ Utsunomiya University, 7-1-2 Yoto, Utsunomiya 321-8585, JAPAN, ${ }^{2}$ JST CREST \\ Fax: 81-28-689-6154, e-mail: katon@cc.utsunomiya-u.ac.jp
}

\begin{abstract}
Expression of some pathogenesis-related genes in gram-negative bacteria is controlled by bacterial cell-to-cell communication system, quorum sensing (QS), of which signal molecule is $\mathrm{N}$-acylhomoserine lactone (AHL). The AHL concentration-dependent prodigiosin production in Serratia marcescens AS-1 as one of model pathogens could be suppressed by trapping AHLs onto cyclic oligosaccharide cyclodextrins (CDs). Adding CDs could suppress the prodigiosin production because the AHL concentration was kept at a low level below a threshold of the QS activation during the culture. The QS inactivation due to formation of the CD-AHL inclusion complex was demonstrated by bioassay of an AHL-synthase defective mutant, Chromobacterium violaceum $\mathrm{CV} 026$, in which purple pigment violacein production could be induced by adding AHLs from outside of cells. A small spot of $N$-hexanoyl-L-homoserine lactone (C6HSL) solution onto an agar gel plate containing $20 \mathrm{wt} \%$ hydroxypropyl- $\beta-\mathrm{CD}$ and CV026 cells resulted in a meaningful reduction of violacein production after a 10-h incubation at $30^{\circ} \mathrm{C}$. A quartz crystal microbalance based on admittance analysis (QCM-A) could determine stability constants between $\beta-\mathrm{CD}$ and C6HSL as $(7 \pm 2) \times 10^{2} \mathrm{M}^{-1}$.

Key words: cyclodextrin, $N$-acylhomoserine lactone, cell-cell communication, bacterial infection
\end{abstract}

\section{INTRODUCTION}

$N$-Acylhomoserine lactones (AHLs) are known as key factors to activate cell-to-cell communication system, quorum sensing (QS) in gramnegative bacteria [1-3]. The local concentration of AHLs outside cells increases with increasing the cell population density. When the concentration reaches a threshold, the expression of QS-dependent gene is activated by AHLreceptor complex because the AHL signals are considered to diffuse outside and inside cells through the cell membrane (Fig.1). After reaching high-cell-density, the diverse biological functions are induced such as bioluminescence, biofilm formation, antibacterial production, etc.

Suppression of Serratia marcescens quorum sensing was focused to develop a novel material for prevention of bacterial infection because some gram-negative pathogens regulate their virulence using the AHL-mediated QS. In recent years, nosocomial infection of antibiotic resistant Serratia marcescens through medical devices was reported. Antibiotic medications are popular ways to kill virulence bacteria. Once some microorganisms acquire the antibiotic resistance, the bacteria can cause serious disease for human. A novel approach for finding feasible solutions is desired to prevent bacterial infection avoiding antibiotic use. However, it has not yet been reported. The authors have been focused on suppression of the QS system because expression of virulence gene, pig cluster and rhlA, respectively is regulated by short-chain AHL mediated QS in Serratia marcescens and Pseudomonas aeruginosa [4-6]. Production of the antibiotic prodigiosin, of which synthesis is regulated by $N$-hexanoyl-L-homoserine lactone (C6HSL) mediated QS [7-8], was investigated to evaluate novel techniques for the QS prevention.

To artificially disturb the QS system, low levels of AHL concentration below the threshold should be maintained. Cyclic oligosaccharide cyclodextrin (CD) was previously reported to form an inclusion complex between its hydrophobic cavity and the acyl chain of AHLs in aqueous solution. Even after CD immobilization on some matrices, the AHL signals could be trapped by host-guest interaction [9-11]. The analyses of ${ }^{1} \mathrm{H}$ NMR and ROESY were carried out to study the AHL complex with $\alpha-$ or $\beta-C D$, of which cavity sizes respectively are 0.45 and 0.70 nm. However, the little is known about the stability constants between CDs and AHL signals.

A $27-\mathrm{MHz}$ quartz crystal microbalance based on admittance analysis (QCM-A) could be applied to determine the stability constants for the $\mathrm{CD}$ complex. Mono (6-deoxy-6- $N, N$-diethylamino)$\beta$-CD could be immobilized onto a gold-surface of the QCM sensor to form the inclusion complex with soluble C6HSL. The conventional QCM measurement in gas phase utilizes a resonance frequency which changes as a linear function of the mass of molecules deposited onto the quartz crystal [12, 13]. Admittance analysis (QCM-A) 


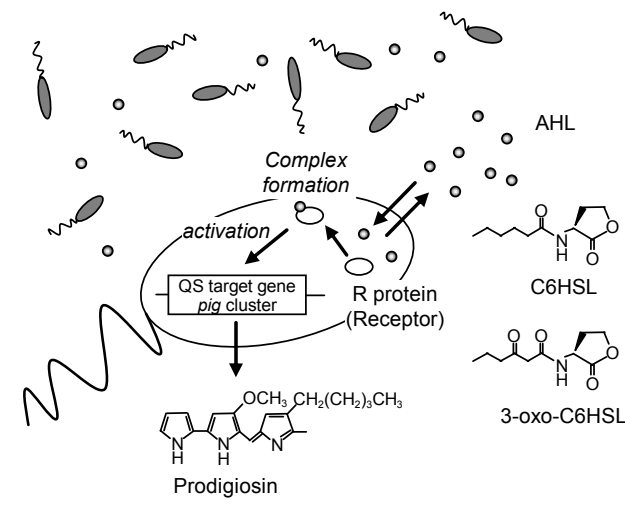

Fig.1 Quorum sensing in Serratia marcescens AS-1.

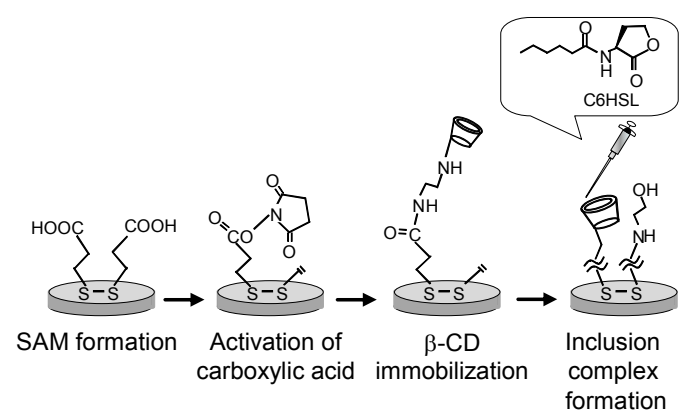

Fig.2 Immobilization of $\beta-C D$ on the gold surface of the QCM sensor plate.

enables to determine the mass change more accurately by using a network analyzer $[14,15]$. A change of resonance frequency $\left(\Delta F_{\mathrm{s}}\right)$ after substrate adsorption can give $\Delta F_{2}$ that allows measurement of the mass load without being affected by the viscous load of a liquid. To determine the stability constants, kinetic analyses were investigated by adding C6HSL onto the CD-modified gold surface of the QCM sensor.

The objectives of this research are to determine the stability constant between the $\beta-C D$ and C6HSL using the QCM-A technique, and also to demonstrate the QS suppression in Serratia marcescens AS-1 due to reducing AHL concentration in the presence of CD hosts.

\section{EXPERIMENTAL}

2.1 Materials

Hydoroxypropyl- $\beta-\mathrm{CD}$ (HP- $\beta$-CD) and 1-ethyl3-(3-dimethylaminopropyl) carbodiimide, hydrochloride (EDC) were purchased from ACROS Organics and Wako Pure Industries, respectively. C6HSL was synthesized from $(S)$ - $\alpha$-amino- $\gamma$-butyrolactone hydrobromide (Aldrich).

\subsection{Prodigiosin production assay}

A pre-culture solution of Serratia marcescens AS-1 was inoculated in Luria-Bertani (LB) medium. After shaking culture at $25^{\circ} \mathrm{C}$ for $24 \mathrm{~h}$, production of red pigment prodigiosin was determined as follows [16]. The culture broth was centrifuged, and then the cell pellet was re-suspended in acidified ethanol to extract the prodigiosin because it was accumulated inside cells. The mixture was centrifuged again to remove cell debris, and the absorbance at $534 \mathrm{~nm}$ of the supernatant $\left(\mathrm{A}_{534}\right)$ was measured by UV-VIS spectrophotometer. The prodigiosin production per cell was calculated as $\mathrm{A}_{534} / \mathrm{OD}_{600}$, while the cell density was given as the turbidity of the culture broth at $600 \mathrm{~nm}\left(\mathrm{OD}_{600}\right)$. To demonstrate the AHL-trapping technique, desired amounts of HP- $\beta$-CD $(0-12 \mathrm{wt} \%)$ was dissolved in the LB medium at the beginning of the shaking culture. HP-modified CD was used because of its high solubility in aqueous media. The inhibitory effects of the CDs on prodigiosin production were investigated after normalization of $\mathrm{A}_{534} / \mathrm{OD}_{600}$.

2.3 QS signal inactivation due to $\mathrm{CD}$ inclusion

Bioassay of Chromobacterium violaceum CV026 was carried out to detect AHLs [17]. The CV026 culture broth was mixed with agar sol at a little high temperature against the gelation point. The CV026 agar gel plates with or without 20 wt $\%$ HP- $\beta-C D$ were prepared in petri dish and then the aqueous solution of $2.5 \mu \mathrm{M}$ C6HSL was spotted to filter papers put on both plates. The production of the purple pigment violacein can be induced only by adding active QS signal molecules from outside cells. Appearance of the purple color was the effective index for the QS activation after incubation for $24 \mathrm{~h}$ at $30^{\circ} \mathrm{C}$.

2.4 Kinetic analysis of the interaction between $\beta-\mathrm{CD}$ and C6HSL using a QCM biosensor

Self-assembled monolayer (SAM) was prepared on the gold surface of the QCM plate using 3, 3'-dithiodipropionic acid. The carboxylic acid groups of the SAM can be converted into the amine-reactive esters by adding $N$-hydroxysuccinimide (NHS) and EDC. Mono (6-deoxy-6- $N$, $N$-diethylamino)- $\beta$-CD was reacted with the esters to induce the $\beta-\mathrm{CD}$ moieties onto the QCM sensor plate. Then ethanolamine was added to deactivate the excess ester groups (Fig.2).

Stabilization of the CD-immobilized sensor in $500 \mu \mathrm{L}$ of distilled water was confirmed by monitoring resonance frequency change $\left(\Delta F_{\mathrm{s}}\right)$ at $20^{\circ} \mathrm{C}$. The $\mathrm{C} 6 \mathrm{HSL}$ aqueous solution $(5-30 \mu \mathrm{L})$ was added to form the inclusion complex with immobilized CDs during stirring, where the initial concentrations of C6HSL were 0.10-1.2 $\mathrm{mM}$. The QCM based on admittance analysis was carried out using Affinix QN Pro (Initium Inc.) equipped with $27 \mathrm{MHz}$ AT-cut quartz-crystal.

The resonance frequency change in a liquid is meaningfully affected by resistance of the viscous liquid at the surface of the QCM's quartz plate, while the mass load is proportional to $\Delta F_{\mathrm{s}}$ described as Sauerbrey equation in the gas phase $[12,13]$. The frequency dependence of the conductance for a quartz resonator allows $\Delta F_{\mathrm{s}}$ to separate into the mass load $\left(\Delta F_{2}\right)$ and the viscous load of a liquid $\left(\Delta F_{\mathrm{w}}\right)$ [14]. Time-dependent $\Delta F_{2}$ 


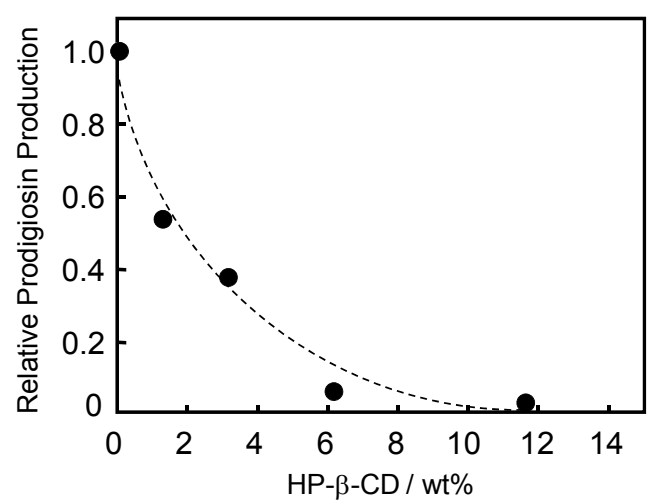

Fig.3 Suppression of the C6HSL-mediated prodigiosin production in $S$. marcescens AS-1 using soluble HP- $\beta$-CDs.

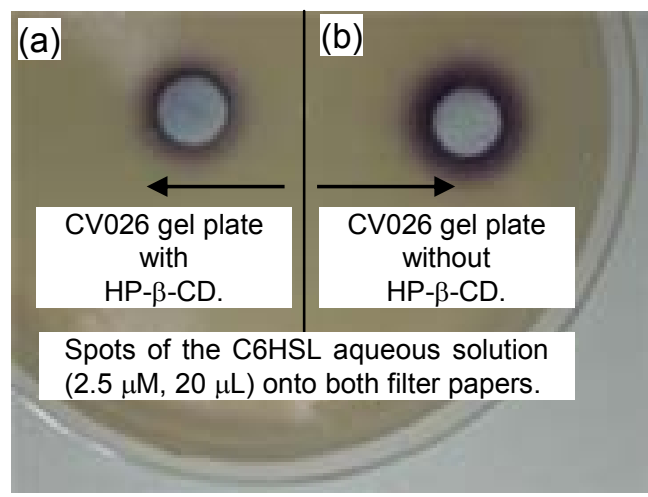

Fig.4 Suppression of QS-dependent violacein production due to inclusion complex formation between added C6HSL and HP- $\beta-C D$. After 10 -h incubation at $30^{\circ} \mathrm{C}$, the purple area was clearly reduced in the presence of $\mathrm{CD}$ hosts.

profiles were reflected only by guest molecule adsorption and then fitted by first-order kinetics equation. The mass sensitivity of the $27 \mathrm{MHz}$ quartz-crystal is set to $30 \mathrm{pg} / \mathrm{Hz}$ by adjusting the surface area of the gold electrode.

\section{RESULTS AND DISCUSSION}

The QS suppression effects of HP- $\beta$-CDs were investigated by measuring amount of red pigment prodigiosin in $S$. marcescens AS-1, of which cell growth was carried out in the LB liquid medium (Fig.3). The relative prodigiosin production decreased with increasing additive CDs, while the $\mathrm{OD}_{600}$ as the cell population density was not affected by coexisting CDs (data not shown). Almost all blocking prodigiosin production in the presence of $12 \mathrm{wt} \%$ HP- $\beta-C D$ suggested that the $\beta-C D$ cavities could capture the acyl chain of the AHLs even in the coexisting of medium components. The $\beta-C D$ could interact enough with short-chain AHL signals for the QS inhibition. It is known that $S$. marcescens AS-1 mainly produces $N$-(3-oxo-hexanoyl)-L-homoserine lactone (3-oxo-C6HSL) and C6HSL as the

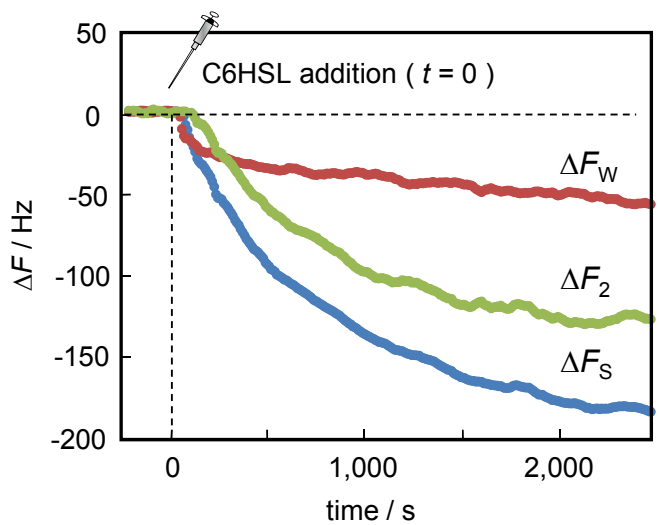

Fig.5 Separation of the resonance frequency change $\left(\Delta F_{\mathrm{s}}\right)$ into two parts: mass load $\left(\Delta F_{2}\right)$ and the viscous load of a liquid $\left(\Delta F_{\mathrm{w}}\right)$.

QS signal molecules and activate pig clusters after the AHL concentration exceeds the threshold, while las QS circuit in $P$. aeruginosa was regulated by long-chain AHL, $N$-(3-oxododecanoyl)-L-homoserine lactone (3-oxo-C12 HSL). The NMR analyses suggested that the $\beta$-CD could form inclusion complex with not lactone ring but acyl chain of the AHL. Cavity sizes of $\alpha$ - and $\beta-C D$ are suitable for $1: 1$ complex with a hydrophobic alkyl chain in aqueous solution.

To demonstrate the QS suppression on solid medium, the AHL assay with $C$. violaceum CV026 as one of the popular AHL reporters was investigated using agar gel plates. The AHLdependent QS system could be activated only by adding AHLs from outside cells because of the AHL synthase gene defect. The purple violacein was observed by adding C6HSL because of the QS activation in CV026 cells (Fig.4b), while violacein production could be clearly suppressed on the agar gel plates containing HP- $\beta-C D$ (Fig.4a). It is known that the QS-dependent violacein production in CV026 strain can be activated only by short-acyl chain AHL [17].

Admittance analysis of the resonance frequency change of the QCM gave $\Delta F_{2}$ as the mass load avoiding effects of the viscous load of a liquid. Immobilized amounts of $\beta-C D$ could be determined as approximately $43 \mathrm{ng} /$ sensor plate by monitoring $\Delta F_{2}$ during the coupling reaction using EDC and NHS. After the excess activated esters were reacted with ethanolamine, the steady-state sensor responses were measured in a static condition. Mass uptake kinetics was studied to obtain the rate constants when the C6HSL was added into a cup-typed QCM sensor cell immobilized with $\beta$-CD. Figure 5 shows a representative result of the $\Delta F$ separation; $\Delta F_{2}$ as the mass load onto the QCM sensor plate was smaller than the resonance frequency change $\left(\Delta F_{\mathrm{s}}\right)$. The change of the resonance frequency was caused by CD-C6HSL interaction because nonspecific adsorption of C6HSL onto the SAM was negligible (data not shown). 


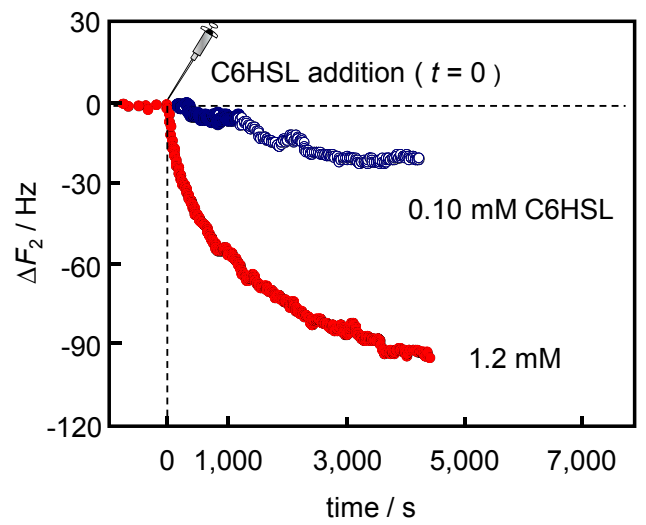

Fig.6 Profiles of $\Delta F_{2}$ with different C6HSL concentrations.

Time profiles of $\Delta F_{2}$ were determined by adding C6HSL as desired concentration (Fig.6), and then the rate constant, $k_{\mathrm{obs}}\left[\mathrm{s}^{-1}\right]$, is obtained by first order curve fitting using Eq. 1 .

$$
\Delta F_{2}=\Delta F_{2, \max }\left[1-\exp \left(-k_{\mathrm{obs}} t\right)\right]
$$

The given rate constant $k_{\mathrm{obs}}$ is described as

$$
k_{\mathrm{obs}}=k_{\mathrm{off}}+[\mathrm{AHL}] k_{\mathrm{on}}
$$

where $k_{\text {on }}$ and $k_{\text {off }}$ are adsorption and desorption rate constants, respectively. To determine the stability constant $K\left(=k_{\text {on }} / k_{\text {off }}\right)$, the intercept $\left(k_{\text {off }}\right)$ and the slope $\left(k_{\mathrm{on}}\right)$ were calculated from a straight line, on which plots of $k_{\mathrm{obs}}$ versus C6HSL concentration fell (Fig.7). The straight line indicates the effective $1: 1$ complex formation between $\beta-C D$ cavity and C6HSL, and their stability constant is given as $K=(7 \pm 2) \times 10^{2}$ $\left[\mathrm{M}^{-1}\right]$. The stability constant between $\beta-\mathrm{CD}$ and 3-oxo-C6HSL was also determined as approximately $K=7 \times 10^{2}\left[\mathrm{M}^{-1}\right]$ (data not shown). These results mean relatively high concentration of the host is required to uptake AHL signals only by hydrophobic interaction onto $C D$ cavities, as compared with that of native receptors. However, CD contents are easily adjusted to realize sufficient effects of QS inhibition when the AHL signals are captured by free or immobilized CDs.

\section{CONCLUSIONS}

By trapping C6HSL onto $\beta-\mathrm{CD}$ cavities, suppression of QS-dependent pigment production could be demonstrated in both AHL synthasedefective mutant $C$. violaceum $\mathrm{CV} 026$ and wild-type $S$. marcescens AS-1. Inclusion complex stability constant of $\beta-\mathrm{CD}$ with $\mathrm{C} 6 \mathrm{HSL}$ was determined as $K=(7 \pm 2) \times 10^{2}\left[\mathrm{M}^{-1}\right]$ by QCM-A analysis. The cyclodextrin host-guest chemistry has high potential to regulate bacterial communication system, and also CD derivatives are one of the useful candidate materials to block AHL-mediated bacterial infection because those are recognized as safe materials for human.

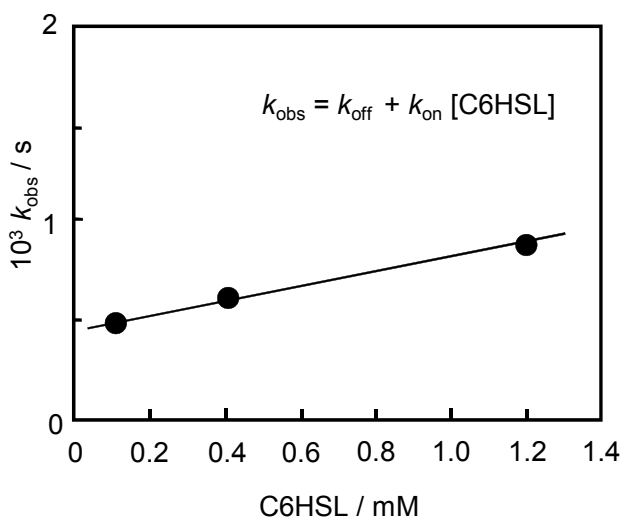

Fig.7 Relationship between $k_{\mathrm{obs}}$ and C6HSL concentration.

\section{REFERENCES}

[1] E. P. Greenberg, ASM News, 63, 371-377 (1997).

[2] C. Fuqua, M. R. Parsek, and E. P. Greenberg, Annu. Rev. Genet., 35, 439-468 (2001).

[3] R. P. Novick, Mol. Microbiol., 48, 1429-1449 (2003).

[4] N. R. Thomson, M. A. Crow, S. J. McGowan, A. Cox, and G. P. C. Salmond, Mol. Microbiol., 36, 539-556 (2000).

[5] A. K. P. Harris, N. R. Williamson, H. Slater, A. Cox, S. Abbasi, I. Foulds, H. T. Simonsen, F. J. Leeper, and G. P. C. Salmond, Microbiol., 150, 3547-3560 (2004).

[6] H. Slater, M. Crow, L. Everson, and G. P. C. Salmond, Mol. Microbiol., 47, 303-320 (2003).

[7] S. A. Dauenhauer, R. A. Hull, and R. P. Williams, J. Bacteriol., 158, 1128-1132 (1984).

[8] A. Hejazi and F. R. Falkiner, J. Med. Microbiol., 46, 903-912 (1997).

[9] N. Kato, T. Morohoshi, T. Nozawa, H. Matsumoto, and T. Ikeda, J. Incl. Phenom. Macro. Chem., 56, 55-59 (2006).

[10] N. Kato, T. Tanaka, S. Nakagawa, T. Morohoshi, K. Hiratani, and T. Ikeda, J. Incl. Phenom. Macro. Chem., 57, 419-423 (2007).

[11] N. Kato, A. Kobayashi, H. Motohashi, Y. Ozonoe, T. Morohoshi, and T. Ikeda, Progr. Colloid Polym. Sci., 136, 155-162 (2009).

[12] K. Kanazawa and J. G. Gordon II, Anal. Chem., 57, 1770-1771 (1985).

[13] S. J. Martin, V. E. Granstaff, and G. C. Frye, Anal. Chem., 63, 2272-2281 (1991).

[14] A. Itoh and M. Ichihashi, Meas. Sci. Technol., 19, 075205, 1-9 (2008).

[15] A. Itoh and M. Ichihashi, Meas. Sci. Technol., 22, 015402, 1-6 (2011).

[16] H. Slater, M. Crow, L. Everson, and G. P. C. Salmond, Mol. Microbiol., 47, 303-320 (2003).

[17] L. Ravin, A. B. Christensen, S. Molin, M. Givskov, and L. Gram, J. Micobiol. Methods, 44, 239-251 (2001).

(Received February 24, 2012; Accepted April 15, 2012) 